\title{
Plant antimicrobial peptides: Possibilities in community health
}

\author{
Durbasree Sarkar ${ }^{1}$, Samput Mallick², Sanjay Maitra ${ }^{3, * *}$ \\ ${ }^{1}$ Student, University of Gour Banga, Malda, West Bengal, ${ }^{2}$ Resident, ${ }^{\mathbf{3}}$ Associate Professor, ${ }^{2,3}$ Dept.of Surgery, Nil Ratan Sircar \\ Medical College and Hospital, Kolkata, West Bengal, India
}

*Corresponding Author:

Email: drsanjaymaitra@gmail.com

\begin{abstract}
Introduction: Anti-microbial peptides (AMPs) are among the first line defense mechanism of a variety of living organisms. The evidence on AMPs has increased considerably during the last two decades, showing they offer more than just antimicrobial activity, and presenting a broad spectrum of physiological functions. Plant AMPs are diverse families of peptides differing in their amino acid structure and composition, displaying broad spectrum antimicrobial activities. AMPs have a high potential for therapeutic use in medical sciences and can be used as alternate to antibiotics, for protection of animals and plants against diseases. Therefore, AMPs are good candidate for their possible beneficial role in public health. The present article reviews the role of plant AMPs in community health setting regarding treatment of cancer, infection control and food safety.
\end{abstract}

Keywords: Antimicrobial peptide, Community health, Host defense peptides.

\section{Introduction}

Anti-microbial peptides (AMPs) are polyamides with gene-encoded 10-50 amino acid residues. They form one of the first lines of defense mechanisms in animals. They are found either in skin or epithelium that form the barriers to bacterial invasion or rapidly delivered to the sites of infection or injury due to bacterial invasion. The AMPs are sub classified into more than ten families. In humans, defensins and cathelicidins, are most important families both produced by neutrophils and epithelial cells. They share similarities in their roles in infection and immunity. Administration of a defensin derived from human neutrophil can reduce bacterial load in infected peritoneal cavity within 24 hours after infection in mice induced with Klebsiella pneumoniae infection. Similarly, cathelicidin-derived peptide LL-37 protects mice from respiratory tularemia due to Francisella tularensis. In mammals, immune systems activate both adaptive and innate immunities. But, lower animals like insects depend almost entirely on a group of AMP against bacteria. At the neutral $\mathrm{pH}$ of 7 , the AMP sequences contain positively charged residues lysine, arginine and histidine, in excess of the negatively charged glutamic and aspartic acids. It appeared that the mechanism of action was accumulation on the negatively charged bacterial membrane surface up to a cut off level beyond which membrane disintegration or disruption occurred. The membrane-peptide interactions depend both on the AMPs and the bacterial cell membrane. For example, magainin, an AMP derived from frog skin, lies in the plane of the bi-layer; while M2 delta, a nicotinic acetylcholine receptor segment, spans the membrane, perpendicular to the bilayer plane. It now seems that the assumptions of the two main membrane-perturbing models (ShaiMatsuzaki-Huang carpet and barrel-stav) for biophysical characteristics are unreliable. The need for alternate models exist because many AMPs fail to modify membrane permeability at concentrations that are microbiologically active. ${ }^{1}$

It was presumed that AMPs act against bacterial infection by disintegrating bacterial membranes. Later, identification of targets within bacteria changed the view toward inhibition of prokaryote-specific biochemical processes. However, the extent of none of these activities account for the efficacy of some of these AMPs in animal models of systemic and cutaneous infections. Recent evidences suggests that many AMPs, now termed as host-defense peptides (HDPs), act by activating the host immune system, recruiting and activating macrophages and mast cells and by inducing chemokine production and altering NF- $\mathrm{kB}$ signaling processes. Consequently, both pro- and antiinflammatory responses are enhanced. Also, there is activation of innate and adaptive immunity mechanisms, wound healing, and apoptosis. HDPs effect on systemic circulation and local injury sites is far more efficient than pure single-endpoint in vitro microbiological or biochemical data would suggest. HDPs enhance tissue repair after or even without bacterial infection. However, the multifaceted and sometimes opposing, immunomodulatory functions of HDPs make their therapeutic use tricky. ${ }^{2}$

Plants produce antimicrobial peptides as an innate defense against pathogens. These are small cysteinerich molecules. On the basis of sequence homology of the amino acids, AMPs were classified mostly as adefensins, thionins, cyclotides, snakins, hevein-like and lipid transfer proteins. Plant antimicrobial peptides are classed into several families based on general features, like mechanism of action targeting outer membrane structures (such as ion channels), overall positive charge, the presence of disulfide bonds (which stabilize the structure). AMPs are appealing for the role in host defence and as simple models for studying the 
molecular mechanism of antimicrobial peptide action. AMPs have the potential to fight pathogens, including those showing increased resistance to conventional antimicrobial compounds. They have broad-spectrum antimicrobial activity against pathogenic fungi and promising agent for managing diseases in sensitive transgenic plants. Many plant AMPs are now well characterized, but their physicochemical and structural information is not properly understood. ${ }^{4}$

The first AMP discovered from plant sources was a purothionin isolated from wheat flour (Triticum aestivum) in 1942. It can inhibit growth of some phytopathogens such as Xanthomonas campestris, Pseudomonas solanacearum and Corynebacterium michiganense. The next peptide in this category was reported 30 years later. In recent years, a number of antimicrobial peptides have been isolated from plant sources. AMPs have been isolated from flowers, roots, stem, seedsand leaves. Plant AMPs illustrated activity towards both phytopathogens and human pathogenic bacteria. As a result, they have become a new tool for the development of methods in the controlling crop loss and for producing newer antibiotics for treatment of variety of human infections. However, knowledge of the mechanism of these peptides to affect the pathogen to cause cellular death or inhibition of growth is still obscure. The fact is that the mode of action is still obscure as only a few peptide structures have been studied. Furthermore, it is unclear if plant AMPs from the different protein families have similar structures, sequences and modes of action, or if the groups behave in a differently. ${ }^{5}$

Food safety is an important public health issue in both developed and developing countries. Illnesses by food borne pathogens are frequent and cause significant morbidity and mortality. Foodborne illnesses caused by foodborne pathogens, such as Salmonella, Vibrio parahaemolyticus, Listeria monocytogenes, Escherichia coli O157: H7, and Shigella, led to a serious public health problem in food safety throughout the world. Foodborne illnesses may cause symptoms of diarrhea or even death. Recent data points to an incidence of up to $61.92 \%$, primarily due to $\mathrm{V}$. parahaemolyticus, Salmonella, E. coli O157:H7, and so on. ${ }^{6}$

Two major economic implications in food safety for the society are bacterial food poisoning and spoiling of food. It is of utmost necessity to develop novel antimicrobial agents to control outbreaks in food supply, in a context of increasing resistance to antibiotics. Spoiling of food and food poisoning caused by bacteria can have major economic and health implications for society. With the rising resistance to conventional antibiotics, there is a need to develop novel antimicrobial agents to combat outbreaks in food supply. Antimicrobial peptides offer potential as novel therapeutics to combat food spoilage and poisoning caused by pathogenic and nonpathogenic bacteria. Some plant defensin-like peptides are effective antimicrobials and may act in concert with HBD3 to control bacteria associated with food spoilage and food poisoning. The peptide human beta-defensin 3 (HBD3) is a potent antimicrobial agent against a wide range of beer-spoiling bacteria. HBD3 is an excellent candidate for development as an additive to prevent food and beverage spoilage. Peptides, which are structurally similar with the human defensin peptide HBD3, may show antimicrobial activity against food-spoiling bacteria. When combined with HBD3, the peptides are highly effective. Two peptides, fabatin-2 and Cpthionin-2, display antimicrobial activity, though these peptides differ from HBD3 being sensitive to salt and thermo-stable. The activities of ultra short peptide like synthetic tetra peptide O3TR, display biphasic antimicrobial activity with a narrower host range than HBD3. The plant defensin-like peptides in pair wise combinations fabatin-2 and $\mathrm{Cp}$-thionin-2 display a synergistic effect with HBD3, while O3TR was antagonistic. $^{7}$

Advances in oncology and transplant have increased survival of immuno compromised patients. This has resulted in increased fungal infections and more frequent resistance to anti fungal drugs due to their increased used. AMPs may provide an alternate in addressing this problem. Murine model of fungal infections show promising results, wherein the potential cytotoxicity of indolicidin for the host cells is reduced by the administration of the formulation in a liposomeencapsulated form. The defensins family has given great hope. Several of these cysteine-stabilized peptides show pronounced activity against fungi. Two peptides, PAF and AFP, were fungicidal to the classes Zygomycetes, Ascomycetes and Basidiomycota. Penicillium brevicompactum vesicle protein inhibited the growth of Saccharomyces cerevisiae. From plants, three peptides have been isolated that have shown activity against Aspergillus spp., Candida albicans, and Neurospora crassa, among others. Most of these peptides were not haemolytic against human erythrocytes $^{8}$ of the various plant derived peptides interest has been generated by lunasin which has anti-neoplastic effects in skin, breast, prostrate, colon, lymphoma and leukaemia cell lines with differing modes of action. In human breast Michigan cancer foundation-7 (MCF-7) cells and murine fibroblast, NIH $3 \mathrm{~T} 3$, lunasin suppresses their transformation, induced by carcinogenic chemicals, by inhibition of histone acetylation in the presence of sodium butyrate. L1210 leukaemic cells exhibit cytotoxic effect, apoptosis through the activation of caspase- 3 and induction of cell cycle arrest in G2/M phase by lunasin. Lunasin induces apoptosis by activating of caspase-3, in human adeno carcinoma HT-29 cell lines, via the intrinsic apoptotic pathway, as demonstrated by induction of $\mathrm{B}$ cell lymphoma 2(Bcl-2) -associated $\mathrm{X}(\mathrm{Bax})$ protein and a reduction in $\mathrm{Bcl}-2$ protein levels. Lunacin can inhibit the metastasis of human colon cancer cells by direct 
binding with $\alpha 5 \beta 1$ integrin, suppressing focal adhesion kinase (FAK)/ERK/NF- $\mathrm{KB}$ signaling. It also potentiates the efficacy of oxaliplatin in prevention of outgrowth of metastasis. In vivo experiments have demonstrated lunacin's anti-carcinogenic actions. In murine models, it has been shown to act against chemically-induced breast cancer, to delay liver metastasis of colon cancer cells and to reduce lymphoma volume. Another plant AMP extracted from rice bran, a pentapeptide, Glutammate-Glycine-Arginine-Proline-Arginine, is known to inhibit the growth of colon cancer cells (Caco-2 and human colorectal adenocarcinoma cell line, HCT-116). It also inhibits the growth of liver cancer cells and breast cancer cells. Anti microbial peptides isolated from legume seeds, the Bowman-Birk inhibitor (BBI), has a well-characterized ability for inhibition of chymotrypsin and trypsin activities and seems to have a preventive effect against prostate, breast and colon cancers by suppression of carcinogenesis (by inducing the tumour suppressor gene connexin 43(Cx43) and cell cycle arrest in the G1/S phase, as in human osteosarcoma cells. BBI elicits a cytotoxic and cytostatic effect in MCF-7 breast cancer cells by apoptosis. It can cross the membrane of breast cancer cells and co-localizes with the proteasome in cytoplasm and nucleus. Lectins recognize specific carbohydrate moieties on malignant cells. Tepary bean (Phaseolus acutifolius) lectins have demonstrated cytotoxic and antiproliferative effects on the human colon carcinoma cell line, Sw480, and cervical cancer cell lines, C33-A. Murine models show that mistletoe derived lectins have an immunomodulatory action in colon carcinoma and prolongs survival in mice with leukaemia cells.

The current progress in plant AMPs is an exciting and growing research field, because amino acid sequence directs all aspects of cellular function and coordinate communications between cells. However, the evidence on bioactive peptides has several limitations till date. Firstly, many AMPs appear to act through multiple mechanisms of action and therefore, to possess pleiotropic activities. Individual components of these should be studied to find out their actions and the specific receptors and signaling pathways involved of their beneficial effects. Secondly, reliable pharmacokinetic study is needed to determine adequate dosage and frequency and routes of administration, analyzing the variability of biological effects. Thirdly, some peptides may need delivery methods for protection from gastrointestinal enzymes when administered orally by variation of dosage forms. Processes of digestion can change the peptide's bioavailability and efficacy turning an apparent bioactive peptide into an inactive one and vice versa.

The specific duodenal peptide transporter mechanism needs further elaboration for understanding bioavailability. Finally, the human factor variability (concomitant therapy, sex, diseases, age, etc.) that influence final efficacy of the peptide, as shown for lactotripeptides, should be considered. So far, the results of in vitro and in vivo studies from plant AMPs are encouraging and shows potential for treatment of numerous diseases or risk factors that have public health impacts. However, there is lacuna of studies in this particular field which demands the attention of experts and scientists.

With further studies, plant AMPs are expected to have impact on a wide range of therapeutic and commercial uses. These will range from anti bacterial agents and antifungal agents to preservatives for food and drinks.

\section{References}

1. Otvos L Jr. Immunomodulatory effects of anti-microbial peptides. Acta Microbiol Immunol Hung. 2016; 63(3):257-277.

2. Zhang L, Falla TJ. Potential therapeutic application of host defense peptides. Methods Mol Biol. 2010;618:30327. doi: 10.1007/978-1-60761-594-1_19.

3. Hammami, R., Ben Hamida, J., Vergoten, G., \& Fliss, I. (2009). PhytAMP: a database dedicated to antimicrobial plant peptides. Nucleic Acids Research, 37(Database issue), D963-D968. http://doi.org/10.1093/nar/gkn655

4. PB Pelegrini, RP del Sarto, ON Silva. Antibacterial Peptides from Plants: What They Are and How They Probably Work. Biochemistry Research International. (2011)

5. Zhao, X., Li, M., \& Xu, Z. Detection of Foodborne Pathogens by Surface Enhanced Raman Spectroscopy. Frontiers in Microbiology, (2018). 9,1236. http://doi.org/10.3389/fmicb.2018.01236doi:10.1155/201 $1 / 250349$

6. Kraszewska, J., Beckett, M. C., James, T. C., \& Bond, U. (2016). Comparative Analysis of the Antimicrobial Activities of Plant Defensin-Like and Ultrashort Peptides against Food-Spoiling Bacteria. Applied and Environmental Microbiology, 82(14), 4288-4298. http://doi.org/10.1128/AEM.00558-16

7. Mirski T, Niemcewicz M, Bartoszcze M, Gryko $\mathrm{R}$, Michalski A. Utilisation of peptides against microbial infections - a review. Ann Agric Environ Med. 2017. 7;25(2):205-210. doi: 10.26444/aaem/74471.

8. Cicero, A. F. G., Fogacci, F., \& Colletti, A. (2017). Potential role of bioactive peptides in prevention and treatment of chronic diseases: a narrative review. British Journal of Pharmacology, 174(11):1378-1394. http://doi.org/10.1111/bph.13608 